\title{
Gastos pre operativos en la Ley del Impuesto a la Renta: Alcances y controversias
}

\author{
Guillermo Hidalgo* \\ Cynthia Aldazábal**
}

En el presente artículo Guillermo Hidalgo y Cynthia Aldazábal comentan la deducción de los gastos pre operativos en la Ley del Impuesto a la Renta. En este se encontrarán las principales vacíos, errores y controversias presentes.

Abogado por la Pontificia Universidad Católica del Perú. Master en administración de empresas por la Adolfo Ibáñez School of Management. Miembro del Instituto Peruano de Derecho Tributario (IPDT) y de la Asociación Fiscal International (IFA). Socio de EY.

** Abogada por la Pontificia Universidad Católica del Perú. Miembro del Instituto Peruano de Derecho Tributario (IPDT) y de la Asociación Fiscal International (IFA). Senior del área de asesoría tributaria de EY. 


\section{Gastos pre operativos en la Ley del Impuesto a la Renta: Alcances y controversias}

Nuestra Ley del Impuesto a la Renta (en adelante, LIR) al igual que muchas otras leyes del mundo que gravan la renta o ganancia de las empresas, contiene regulaciones específicas acerca de lo que se conoce como gastos pre operativos, es decir, aquellos gastos incurridos por las empresas con anterioridad al inicio de sus operaciones.

El objeto de este artículo es analizar las reglas que, sobre este tópico, tiene nuestra LIR y su Reglamento; hacer un breve repaso de su tratamiento bajo las normas contables, y; así finalizar con el análisis de los principales vacíos y controversias que suscita nuestra normativa.

\section{Definición general y regulaciones en la Ley del Impuesto a la Renta en el Perú.}

Los desembolsos que efectúa una empresa durante la etapa previa al inicio de sus actividades productivas - que son las que potencialmente le permitirán la generación de rentas gravadas en el futuro - suelen tener un tratamiento tributario particular en los ordenamientos jurídicos atendiendo a la naturaleza de los mencionados desembolsos.

En efecto, habría que anotar que se trata de gastos que, de ser incurridos por una entidad operativa, afectarían la determinación de la renta neta tributaria en el periodo en que se incurren. Lo que ocurre con los gastos pre operativos es que en el momento en que se incurren, no se encuentran vinculados con la generación de ingresos, pues la entidad que los incurre aún no se encuentra generando rentas.

Es así que en la legislación comparada los ordenamientos tributarios dan la posibilidad de diferir este tipo de gastos y deducirlos en un periodo posterior al cual fueron incurridos ${ }^{1}$.

En la legislación chilena, el Decreto Ley 824 del 31 de diciembre de 1974 regula los gastos pre operativos bajo el concepto de "gastos de organización y puesta en marcha" en el numeral 9 del Artículo 31 de la Ley sobre Impuesto a la Renta, el cual establece la deducibilidad de:

«Los gastos de organización y puesta en marcha, los cuales podrán ser amortizados hasta en un lapso de seis ejercicios comerciales consecutivos contados desde que se generaron dichos gastos o desde el año en que la empresa comience a generar ingresos 
Ello tiene su base en el concepto de asociación de costos y gastos establecido por la Norma Internacional de Contabilidad (en adelante, NIC) 1, según el cual para efectos de la aplicación del devengado es indispensable vincular directamente el ingreso que figura en el estado de resultados con todos los gastos en los que se incurre para la generación de dicho ingreso, por existir una relación causa - efecto entre gastos e ingresos.

En este contexto, nuestra LIR, aprobada por el Decreto Legislativo 774 prescribe sobre el tratamiento de los gastos pre operativos en el inciso g) del Artículo 37, y las normas reglamentarias están en el inciso d) del Artículo 21 del reglamento de dicha Ley, aprobado por el Decreto Supremo 122-94-EF (en adelante, el Reglamento) ${ }^{2}$.

El inciso g) del Artículo 37 de la LIR establece lo siguiente:

«Los gastos de organización, los gastos pre operativos iniciales, los gastos pre operativos originados por la expansión de las actividades de la empresa y los intereses devengados durante el periodo pre operativo, a opción del contribuyente, podrán deducirse en el primer ejercicio o amortizarse proporcionalmente en el período máximo de diez (10) años»

A su vez, el inciso d) del Artículo $21^{\circ}$ del Reglamento establece lo siguiente:

«La amortización a que se refiere el inciso g) del Artículo 37 de la Ley, se efectuará a partir del ejercicio en el que se inicie la producción o explotación.

Una vez fijado el plazo de amortización sólo podrá ser variado previa autorización de la SUNAT. El nuevo plazo se computará a partir del

de su actividad principal, cuando este hecho sea posterior a la fecha en que se originaron los gastos».

Así también, en la legislación argentina el Impuesto a la Ganancias (análogo a nuestro IR) cuyo texto único ordenado fue aprobado por el Decreto 649/97 del 06 de agosto de 1997, regula los "gastos de organización" en el inciso c) de su Artículo 87 como una de las deducciones especiales de la tercera categoría (renta empresarial), estableciendo que: «la Dirección General Impositiva admitirá su afectación al primer ejercicio o su amortización en un plazo no mayor de cinco años, a opción del contribuyente».

2 El texto actual del inciso g) del Artículo 37 de la LIR, aprobada por el Decreto Legislativo 774, es el mismo desde la LIR aprobada por la Cuarta Disposición Final de la Ley No 25381 (vigente desde enero de 1992). Así también, el texto actual del inciso d) del Artículo 21 del Reglamento de la LIR aprobado por el Decreto Supremo 122-94-EF no ha sufrido modificaciones a lo largo de los años. Cabe mencionar que anteriormente a dicha disposición, el Reglamento de la Ley 25381 aprobado por el Decreto Supremo 068-92-EF (vigente desde abril de 1992) regulaba prescripciones sobre los gastos pre operativos similares a las vigentes actualmente. 
ejercicio gravable siguiente a aquél en que fuera presentada la solicitud por el contribuyente, sin exceder en total el plazo máximo de 10 años.

Los intereses devengados durante el periodo pre operativo comprenden tanto a los del periodo inicial como a los del periodo de expansión de operaciones de la empresa».

De una lectura de las disposiciones que regulan este tipo de gastos en el Perú podemos señalar lo siguiente:

Nuestra legislación comprende a los gastos de organización, los gastos pre operativos iniciales y a los gastos pre operativos de expansión, así como los intereses devengados en ambos periodos.

Se desprende de ello, que no sólo están comprendidos los gastos de organización - entendidos éstos como los gastos administrativos y legales de constitución de la entidad - sino que además se incluye:

1) Cualquier gasto que se incurra en el periodo pre operativo inicial (luego de la constitución de la entidad pero antes del inicio de operaciones). Debe tratarse de un gasto efectivo, y no de un costo. Tal sería el caso de de las erogaciones por mano de obra para la construcción de un activo fijo efectuadas durante el periodo pre operativo, las cuales deben afectar el costo del activo fijo según lo previsto expresamente por el Artículo 21 de la Ley del IR, $y$, en consecuencia, no califican como gasto pre operativo. ${ }^{3}$

2) Los intereses por financiamientos incurridos durante el periodo pre operativo inicial (antes del inicio de las actividades).

3) Los gastos devengados una vez iniciadas las operaciones, pero asociados con la expansión de las actividades de la empresa.

4) Los intereses por financiamientos incurridos durante el periodo pre operativo de expansión para dicho propósito, que se devenguen durante tal periodo.

Los gastos anotados en el punto anterior podrán, a opción del contribuyente: 1) deducirse en el primer ejercicio que debe entenderse como el primero en que

3 Igual ocurre en el caso de contratos de construcción, los cuales, según el criterio recogido por el Tribunal Fiscal en la Resolución 591-4-2008 califican como costo del contrato y no como gastos pre operativos. 
se inicia la producción o explotación ${ }^{4} ;$ o, 2) deducirse proporcionalmente hasta en un periodo máximo de 10 años.

Hay que notar que nuestra legislación, al reconocer la deducción de los gastos pre operativos hasta el periodo en que se inician operaciones, recoge plenamente el criterio de asociación establecido por la NIC 1 citada anteriormente, tal como lo ha señalado la Resolución del Tribunal Fiscal (en adelante, Resolución del Tribunal Fiscal) 2989-4-2010 5 .

En ambos casos 1) y 2) señalados en el primer párrafo del punto inmediato anterior, la deducción se encuentra condicionada al inicio de la producción o explotación del negocio.

Cabe precisar también que si el contribuyente opta por la amortización en el plazo de hasta 10 años, la deducción de dicha amortización debe ser proporcional; es decir, en partes iguales desde el inicio de operaciones hasta que se agote.

Hay que notar que el concepto de gasto pre operativo no se encuentra relacionado a la vida útil de un bien, como sucede con la depreciación de los activos fijos. En ese sentido, si el inicio de operaciones ocurrió, por ejemplo, en noviembre del ejercicio 2012 y el contribuyente optó por la amortización en 10 años, en el ejercicio 2012 podrá deducir como gasto pre operativo vía amortización un décimo $(1 / 10)$ del total del gasto pre operativo y no solamente el proporcional a los dos meses transcurridos desde el inicio de operaciones, a diferencia de lo que ocurre con la depreciación.

Al respecto, la Resolución del Tribunal Fiscal 5349-3-2005 señaló que:

«[...] la amortización a que se refiere el inciso g) del Artículo 37 de la IR, deberá efectuarse a partir del ejercicio en el que se hubiera iniciado la producción o explotación, entendiéndose por ejercicio al

Al respecto el Informe 062-2009-SUNAT-/2B0000 señala que: «La opción de deducir en el primer ejercicio los gastos de organización y pre-operativos contenida en el inciso g) del Artículo $37^{\circ}$ del TUO de la IR debe entenderse referida a la deducción de dichos gastos en el ejercicio gravable en que se inicie la producción o explotación», criterio que compartimos.

5 La referida resolución señala que los lineamientos de la LIR y su reglamento en relación con los gastos pre operativos guardan relación con los postulados contables, « [...] dado que permiten correlacionar los gastos pre operativos con los ingresos generados al momento de haber incurrido en ellos, siendo necesario establecer, aun cuando dichas normas no lo indiquen expresamente, un control mediante el cual se lleve a cabo el registro, valuación y amortización de los gastos pre operativos». 
periodo que se inicia el 1 de enero de cada año y que finaliza el 31 de diciembre del mismo año y proporcionalmente, esto es, en partes iguales entre los años del plazo que el contribuyente optó para efecto de la amortización.

Que lo antes expuesto se sustenta en la propia naturaleza de los desembolsos efectuados, que como gastos diferidos, a diferencia de los activos fijos tangibles, su asignación no se encuentra relacionada con la vida útil de un activo tangible, no siendo por tanto razonable entender que la proporcionalidad de la amortización a la que alude el precitado inciso g) del Artículo 37 de la Ley del IR obedezca al reconocimiento de los beneficios económicos obtenidos durante dicha vida útil».

Cabe anotar que existen normas especiales que establecen un tratamiento diferenciado para algunos gastos pre operativos incurridos en ciertas actividades productivas. Tal es el caso por ejemplo, de los gastos de exploración y desarrollo incurridos por las empresas mineras, regulados por el Artículo $74^{\circ}$ del Decreto Supremo 14-92-EM, Texto Único Ordenado de la Ley General de Minería ${ }^{6}$, así como los gastos de exploración y desarrollo incurridos por las empresas de hidrocarburos, regulados por el Artículo 53 de la Ley 26221, Ley Orgánica de Hidrocarburos ${ }^{7}$.

6 De acuerdo con la referida disposición «el valor de adquisición de las concesiones, se amortizará a partir del ejercicio en que de acuerdo a ley corresponda cumplir con la obligación de producción mínima, en un plazo que el titular de actividad minera determinará en ese momento, en base a la vida probable del depósito, calculada tomando en cuenta las reservas probadas y probables y la producción mínima obligatoria de acuerdo a ley. El plazo así establecido deberá ser puesto en conocimiento de la Administración Tributaria al presentar la Declaración Jurada Anual del Impuesto a la Renta correspondiente al ejercicio en que se inicie la amortización, adjuntando el cálculo correspondiente.

El valor de adquisición de las concesiones incluirá el precio pagado, o los gastos de petitorio, según el caso.

Igualmente, incluirá lo invertido en prospección y exploración hasta la fecha en que de acuerdo a ley corresponda cumplir con la producción mínima, salvo que se opte por deducir lo gastado en prospección y/o exploración en el ejercicio en que se incurra en dichos gastos. Cuando por cualquier razón la concesión minera fuere abandonada o declarada caduca antes de cumplir con la producción mínima obligatoria, su valor de adquisición se amortizará íntegramente en el ejercicio en que ello ocurra. En el caso de agotarse las reservas económicas explotables, hacerse suelta o declararse caduca la concesión antes de amortizase totalmente su valor de adquisición; podrá, a opción del contribuyente, amortizarse de inmediato el saldo, o continuar amortizándose anualmente hasta extinguir su costo dentro del plazo originalmente establecido.»

7 El Artículo 53 de la Ley Orgánica de Hidrocarburos establece que: «Los gastos de exploración y desarrollo así como las inversiones que realicen los Contratistas hasta la fecha en que se inicie la extracción comercial de Hidrocarburos, incluyendo el costo de 
En efecto, las normas de la LIR establecen reglas generales para los gastos pre operativos, mientras que las normas citadas de la Ley General de Minería y Ley Orgánica de Hidrocarburos establecen reglas especiales para los gastos incurridos en la etapa pre operativa que califiquen como gastos de exploración o desarrollo bajo las referidas normas sectoriales.

Sin perjuicio de ello, los gastos incurridos antes del inicio de las operaciones de las empresas mineras o petroleras o incurridos con motivo de la expansión de sus actividades, que no se encuentren alcanzados por las disposiciones especiales antes citadas, ni que califiquen como costos del proyecto o activos fijos, deberían ceñirse al tratamiento general establecido por el inciso g) del Artículo $37^{\circ}$ de la Ley del IR. Tal sería el caso, por ejemplo, de los gastos de constitución de la empresa minera o petrolera, los cuales no califican como gastos de exploración o desarrollo por lo que no se encuentra regulados por las normas especiales antes citadas.

\section{Tratamiento previsto por las normas contables.}

Para propósitos contables, los gastos pre operativos se encuentran regulados en el párrafo 69 de la Norma Internacional de Contabilidad 38 "Activos Intangibles". La referida norma contable establece que cuando se incurren en desembolsos relacionados con la generación de beneficios futuros pero que no implican la adquisición o creación de un activo tangible o intangible, los referidos desembolsos deben tratarse como gasto en el periodo en que se incurren. Se menciona, entre otros ejemplos de esta situación, a los gastos de puesta en marcha de operaciones como sigue:

«a) Gastos de puesta en marcha de operaciones, salvo que las partidas correspondientes formen parte del costo del elemento de propiedades planta y equipo, siguiendo lo establecido en la NIC16. Los gastos de puesta en marcha pueden incluir los costos legales y administrativos para la creación de la entidad jurídica, desembolsos

los pozos, serán acumulados en una cuenta cuyo monto, a opción del Contratista y respecto de cada Contrato, se amortizará de acuerdo con cualesquiera de los dos métodos o procedimientos siguientes:

a) En base a la unidad de producción; o,

b) Mediante la amortización lineal, deduciéndolos en porciones iguales, durante un período no menor de cinco (5) ejercicios anuales.

Iniciada la extracción comercial se deducirá como gasto del ejercicio, todas las partidas correspondientes a egresos que no tengan valor de recuperación $[\ldots]$ » 
para abrir una nueva entidad o comenzar una nueva operación o costos de lanzamiento de nuevos productos o procesos» ${ }^{8}$

Como se puede apreciar, bajo la referida la norma contable los gastos de puesta en marcha de la entidad (gastos de organización o gastos pre operativos iniciales), así como los gastos de lanzamiento de nuevos productos (análogos a los gastos pre operativos de expansión) deben afectar los resultados en el ejercicio en que se incurren. Ello, a diferencia del tratamiento tributario señalado en la sección 1 de este Artículo.

En este sentido, la diferencia entre el tratamiento contable y tributario generará diferencias de carácter temporal que deberán ser conciliadas en la declaración jurada 9 .

Cabe preguntarse si actualmente existe justificación para un tratamiento contable distinto del tributario. El análisis que debería hacerse para arribar a una conclusión sobre este punto requeriría desviarnos del objeto de este Artículo. Baste decir que si el propósito de las normas contables es, entre otros, mostrar la verdadera situación financiera de la empresa, lo que incluye su resultado contable, salvo que se justifique por razones de control fiscal, no debieran existir diferencias entre el tratamiento contable y el tratamiento tributario.

Por otro lado, hay que notar que la norma contable citada no dice nada acerca de los intereses incurridos en el periodo operativo. Ello, pues el tratamiento de tales intereses se encuentra regulado en la Norma Internacional de Contabilidad 23, la cual prescribe, en líneas generales, que los intereses deben afectar el costo del activo fijo si es que son atribuibles a la adquisición, producción o construcción de un activo "calificado". En caso contrario, de no estar asociados a un activo calificado, los intereses afectan los resultados del periodo. Hay varios criterios contables para calificar a un activo como "calificado" para la activación de los intereses. Brevemente, con la finalidad de no desviarnos del objeto central de este Artículo, un activo calificado será un activo significativo para el negocio de la entidad y fabricado "a la medida", por ejemplo la

$8 \quad$ La NIC 38 citada es la versión revisada del 31 de marzo de 2004. Mediante la Resolución del Consejo Normativo de Contabilidad 053-2013 se oficializa le versión 2013 de las Normas Internacionales de Información Financiera (NIC, NOF, CGINIF y SIC).

9 Es decir, si el contribuyente opta por amortizar los gastos pre operativos en un período máximo de 10 años, deberá adicionar dicho gasto en la determinación del IR en el ejercicio en que estos ocurran para luego deducirlos proporcionalmente en los ejercicios siguientes vía Declaración Jurada Anual del IR. 
construcción de una planta termoeléctrica. En ese caso, los intereses deben afectar el costo del activo, y no los resultados, no haciendo distinción a este tratamiento el hecho que la entidad se encuentre en un periodo pre operativo $o$ que ya haya iniciado operaciones. Como se puede apreciar, este tratamiento contable es radicalmente distinto al que prevén las normas tributarias para el caso de los intereses incurridos en el periodo pre operativo inicial tanto como el de expansión, lo cual, a su vez, también generaría diferencia temporales para el cálculo del Impuesto a la Renta ${ }^{10}$.

\section{Algunos temas controvertidos en la regulación peruana de los gastos pre operativos.}

En esta sección analizaremos algunos asuntos controvertidos en nuestro ordenamiento jurídico respecto al tratamiento de los gastos pre operativos:

\subsection{Alcance del término "expansión de actividades".}

Como se señaló anteriormente, de conformidad con el inciso g) del Artículo 37 de la Ley del IR antes citado, los gastos pre operativos no sólo incluyen los gastos incurridos con motivo del inicio de actividades sino también aquellos destinados a "la expansión de las actividades de la empresa". Al respecto, ni la Ley del IR ni su reglamento han establecido una definición de lo que debe entenderse como "expansión de actividades", con lo que ha surgido una controversia sobre los alcances de dicho término.

Al respecto, la Norma IX del Título Preliminar del Código Tributario establece que en lo no previsto en las normas tributarias, como es el caso, podrán aplicarse normas distintas a las tributarias siempre que no se les opongan ni las desnaturalicen. Supletoriamente se aplicarán los Principios del Derecho Tributario, o en su defecto, los Principios del Derecho Administrativo y los Principios Generales del Derecho.

No obstante ello, no hemos identificado en normas distintas a las tributarias una definición de "expansión de actividades". En ese sentido, acudimos al Diccionario de la Real Academia de la Lengua Española, según el cual, expansión es «la acción y efecto de extenderse o dilatarse». A su vez, se define extenderse, entre otras acepciones, como «hacer que algo, aumentando su superficie, ocupe más lugar 0 espacio que el que antes ocupaba»

10 Más aún, el Artículo 20 de la Ley del IR establece que «en ningún caso los intereses formarán parte del costo computable». 
o «dar mayor amplitud y comprensión que la que tenía a un derecho, una jurisdicción, una autoridad, un conocimiento, etc» ${ }^{11}$.

Si bien la definición del referido diccionario está en un contexto diferente al que nos ocupa, es posible notar que es bastante amplia respecto al alcance que podría tener el término "expansión" y el que podría tener "expansión de actividades", pudiendo comprender ésta tanto los gastos incurridos con motivo de la realización de una nueva actividad por parte de la empresa - por ejemplo, una empresa embotelladora de bebidas gaseosas que decide incursionar en la fabricación y venta de cerveza, que es otro producto- como los gastos incurridos para ampliar la propia actividad que la empresa viene realizando -, por ejemplo la misma empresa embotelladora que decide incursionar con los mismos productos que viene fabricando en nuevos mercados en el Perú o en el extranjero.

El Tribunal Fiscal ha emitido pronunciamientos sobre ambas acepciones de lo que podría entenderse por "gastos de expansión".

En efecto, respecto a la realización de una nueva actividad, el Tribunal Fiscal mediante la Resolución del Tribunal Fiscal 971-1-2006 se ha pronunciado sobre una compañía dedicada al servicio de hotelería y campamentos, que realiza desembolsos a efectos de emprender también la actividad de fabricación de productos químicos hoteleros (tales como amenities, productos de limpieza industrial, suavizante de ropa, perfumador ambiental, entre otros) señalando que:

«[...] es preciso mencionar que la norma antes expuesta permite la deducción de gastos de investigación, desarrollo o pre operativos vinculados con la expansión de las actividades de las empresas, es decir, que tienen por objeto lograr un nuevo producto o servicio, los cuales resultan necesarios para determinar si la inversión será adecuada o favorable para sus intereses, pero no condiciona tal deducción a la efectiva generación de ingresos, pues lo relevante es su potencialidad para generar beneficios futuros en el entendido que la inversión puede no ser exitosa» (el subrayado es nuestro).

Así también, la Resolución del Tribunal Fiscal 3942-5-2010 señaló que:

«Que la Administración observó que la recurrente adquirió vía contrato de leasing una máquina sopladora modelo Blow 6 de Gerosa que sirve para soplar botellas de plástico para gaseosas, actividad no desarrollada por la empresa en el ejercicio 2001 y parte del 2002,

11 Real Academia Española, Diccionario de la lengua Española/ Real Academia Española", 2013, http://rae.es/. [Consulta: viernes, 26 de setiembre de 2013] 
entendiéndose que con esta adquisición estaría expandiendo sus actividades.

[...] Que obra en autos de la Factura 001-021930 por la producción de soplado correspondiente al periodo de diciembre de 2002, con lo cual se acredita que es recién en dicho periodo que la recurrente inicia esta nueva línea de negocio [...]

Que de lo expuesto se tiene que los gastos financieros generados en etapa pre-operativa por la adquisición de la máquina sopladora Modelo Blow 6 de Gerosa fueron incurridos por la recurrente con la finalidad de expandir sus actividades económicas[...]» (el subrayado es nuestro).

De la misma manera, la Resolución del Tribunal Fiscal No 591-4-2008 sigue un criterio similar a la Resolución del Tribunal Fiscal anterior indicando que:

«Los desembolsos realizados para efecto de la elaboración de las propuestas técnicas para la preclasificación como postor y para la propuesta como postor para licitación materia de análisis calificarían como gastos pre operativos por cuanto estos han sido incurridos a efecto de postular a una licitación y posteriormente, de obtenerse la buena pro, ejecutar la obra, siendo que los mismos se encuentran claramente vinculados con su actividad gravada» (el subrayado es nuestro).

Por otro lado, el Tribunal Fiscal también ha calificado como gastos de expansión de actividades a aquellas erogaciones destinadas a la ampliación del mercado con el mismo producto. En cuanto a ello, citamos la Resolución del Tribunal Fiscal 10167-2-2007 referente a una empresa que fabrica y vende colchones en el mercado nacional e incurre en gastos para la inscripción de sus marcas de fábrica en el extranjero dado que tiene la intención de empezar a exportar sus productos:

«[...] la inscripción de una marca no sólo se efectúa con la finalidad de diferenciar en el mercado los productos y servicios de una persona de los productos o servicios de otra persona, sino que confiere a su titular el derecho de actuar contra cualquier tercero que sin su consentimiento realice actos destinados al aprovechamiento indebido de la marca generada y que, en caso, de las futuras exportaciones de sus productos, el referido inciso g) del Artículo 37 de la Ley del Impuesto a la Renta permite la deducción de gastos pre operativos vinculados con la expansión de las actividades de las empresas, pero no condiciona tal deducción a la efectiva generación de ingresos, pues lo relevante es su potencialidad para generar 
beneficios futuros en el entendido que la inversión puede no ser exitosa $[. .$.$] » (el subrayado es nuestro).$

Así también, la Resolución del Tribunal Fiscal 05349-3-2005, recoge el caso de una empresa dedicaba al suministro de agua potable que efectúa desembolsos con la finalidad de contar con nuevas fuentes y sistema de recolección de agua, siendo que califica a dichas erogaciones como gastos de expansión. Dicha resolución establece expresamente que:

«[...] Los desembolsos realizados por los estudios y proyectos (nuevas fuentes de agua, sistemas de recolección de agua de lluvias y proyecto especial cono norte) materia de análisis de la empresa Sedapar S.A. califican como gastos diferidos por cuanto estos han sido incurridos a efecto de beneficiar ejercicios posteriores y que para efecto de sus deducción debían encontrarse asociados con los ingresos obtenidos por los mismos. Es decir, estos gastos son calificados como gastos pre operativos a pesar que la empresa ya se dedicaba al suministro de agua» (el subrayado es nuestro).

Como se puede apreciar, el Tribunal se ha pronunciado en el sentido que los gastos pre operativos comprenden el desarrollo de una línea nueva de negocio, pero también la ampliación de las mismas actividades en nuevos mercados.

Como indicamos anteriormente, semánticamente hablando el concepto de "expansión" es amplio, por lo que no encontramos sustento legal válido para restringir los alcances de "la expansión de actividades" solamente a los casos en los que se implementa un nuevo producto o servicio no realizado anteriormente. Más aún, atendiendo a que el sentido de las normas que regulan los gastos pre operativos iniciales o de expansión es recoger el criterio de asociación de ingresos con gastos, resulta lógico entender que dicho criterio debiera comprender a cualquier gasto de "expansión", ya sea que éste se genere por la incorporación de un nuevo giro de negocio, o por el incremento en el volumen del negocio actual, pues en ambos casos los ingresos que serían generados por dicha "expansión" ocurrirían en un periodo posterior a aquel en que se incurrieron los gastos necesarios para generarlos.

Sin perjuicio de ello, dados los significados que puede comprender el concepto bajo comentario, sería importante que, ya sea por la vía reglamentaria o mediante una jurisprudencia de observancia obligatoria ${ }^{12}$ se establezca que la

12 El Artículo 54 del Código Tributario establece que «las Resoluciones del Tribunal Fiscal que interpreten de modo expreso y con carácter general el sentido de normas tributarias, las emitidas en virtud del Artículo $102^{\circ}$, así como las emitidas en virtud a un criterio recurrente de las Salas Especializadas, constituirán jurisprudencia de observancia obligatoria para los órganos de la Administración Tributaria, mientras dicha interpretación 
expansión de actividades comprende tanto la realización de nuevas actividades no realizadas anteriormente, como la de aquellas que ya se vienen realizando.

\subsection{Gastos sujetos a límite incurridos en el periodo pre operativo.}

La LIR sujeta la deducibilidad de algunos tipos de gastos a ciertos límites cuantitativos, como por ejemplo el nivel de ingresos, el valor del patrimonio, etc. Este tipo de limitación responde a razones de control fiscal en ciertos casos en los que se quiere evitar abusos en la deducción de los gastos y se impone por Ley un límite que pretende establecer un criterio de razonabilidad para la deducción de éstos.

Tal es el caso, por ejemplo, del inciso q) del Artículo $37^{\circ}$ de la LIR, que establece que los gastos de representación sólo serán deducibles hasta el $0.5 \%$ de los ingresos brutos de la Compañía con un límite máximo de 40 UITs. A su vez, el inciso II) del Artículo antes citado señala que los gastos recreativos serán deducibles siempre que no excedan del $0.5 \%$ de los ingresos netos del ejercicio hasta el límite de 40 UITs. Igualmente, el inciso a) de la disposición legal mencionada dispone que los intereses por concepto de los préstamos provenientes de partes vinculadas serán deducibles en la medida que el préstamo no supere tres veces el patrimonio del deudor.

Por otro lado, el literal m) del Artículo 37 de la Ley del IR dispone que las dietas de directorio serán deducibles en la medida que no superen el $6 \%$ de la utilidad comercial del ejercicio antes del Impuesto a la Renta.

Al respecto, podría ocurrir que en la etapa pre operativa se incurran en este tipo de gastos, en cuyo caso cabría preguntarse si:

- Estos gastos, incurridos durante el periodo pre operativo, ¿se rigen por la regla de gastos pre operativos del inciso g) del Artículo 37 antes citado; o, al estar regulados en otros incisos del propio Artículo 37, deben regirse por lo dispuesto en éstas normas, por aplicación de un principio de especialidad? En tal caso, los gastos serían deducibles, siempre que en cada caso cumplan con los límites previstos en las normas correspondientes, en el ejercicio en que se incurren ${ }^{13}$. Cabe advertir que bajo dicha disposición es muy probable

no sea modificada por el mismo Tribunal, por vía reglamentaria o por Ley. En este caso, en la resolución correspondiente el Tribunal Fiscal señalará que constituye jurisprudencia de observancia obligatoria y dispondrá la publicación de su texto en el Diario Oficial [...]». 
que los referidos gastos nunca resulten deducibles, pues por el propio hecho que aún no se inician operaciones, no existirían ingresos o resultados positivos que sustenten los límites.

- De recibir dichos gastos el tratamiento de los gastos pre operativos, deberían deducirse, a opción del contribuyente en el ejercicio en que se inician operaciones, 0 amortizarse en los ejercicios siguientes con un máximo de 10 años. En tal caso, ¿no serían aplicables los límites dispuestos por las normas especiales para su deducibilidad? O, de ser aplicables los referidos límites, ¿en qué ejercicio debieran evaluarse dichos límites aplicables para determinar el importe deducible? ¿Cuándo el gasto se incurrió - en cuyo caso estaríamos en el escenario descrito en el punto anterior - o cuando éste se deduce efectivamente?

Siendo éste un tema crucial particularmente para el caso de proyectos complejos con altos niveles de inversión y largos periodos de maduración - por ejemplo, empresas mineras, petroleras o hidroeléctricas - es importante una definición a nivel legislativo o jurisprudencial sobre este asunto.

\subsection{Ganancias o pérdida por diferencia en cambio incurrida en el periodo pre operativo.}

Otro tema que podría suscitar dudas es el referente al tratamiento tributario que se le debe otorgar a la ganancia o pérdida por diferencia en cambio generada en la etapa pre operativa del negocio.

En efecto podría pensarse que la pérdida en cambio incurrida en el periodo pre operativo debe regularse según lo dispuesto por el inciso g) del Artículo 37 de la LIR, esto es como un gasto pre operativo.

Al respecto, creemos más bien que dada su naturaleza, la diferencia en cambio debe afectar los resultados del periodo de conformidad con lo dispuesto en el Artículo 61 de la LIR.

En efecto, la diferencia en cambio, sea positiva o negativa no tiene naturaleza de ingreso o gasto, sino que más bien es un incremento o pérdida patrimonial,

directorio, en el que se estableció que «para que sean deducibles las dietas de directorio no es necesario que se hayan pagado antes del plazo establecido para la presentación de la declaración jurada anual del ejercicio respectivo, ya que el requisito previsto en el inciso v) del Artículo 37 de la LIR no es aplicable a los gastos deducibles contemplados en los otros incisos del referido Artículo 37 que constituyan para su perceptor rentas de segunda, cuarta y quinta categorías». El mismo criterio ha sido recogido en las Resoluciones del Tribunal Fiscal 7014-1-2005, 7719-4-2005 y 20790-1-2012. 
según corresponda. Es por ello que su tratamiento para propósitos impositivos se encuentra previsto en el Artículo 61 de la LIR el cual señala que como regla general en los incisos b), c) y d) las diferencias en cambio se consideran como ganancia o pérdida en el periodo en que se generan. ${ }^{14}$

Así lo ha establecido el Tribunal Fiscal en reiteradas resoluciones. ${ }^{15}$

En este sentido, la diferencia en cambio incurrida en el periodo pre operativo, sea positiva o negativa, debe afectar los resultados del periodo en el que ésta se genera.

Cabe advertir que hasta el ejercicio 2012 estuvo vigente el inciso f) del Artículo 61 de la LIR, según el cual la diferencia en cambio originadas por pasivos en moneda extranjera relacionadas con activos fijos existentes o en tránsito u otros activos permanentes a la fecha del balance general, debía afectar el costo del activo. Sin embargo, dicha disposición legal fue derogada por el Decreto

14 «Artículo $61^{\circ}$.- Las diferencias de cambio originadas por operaciones que fuesen objeto habitual de la actividad gravada y las que se produzcan por razones de los créditos obtenidos para financiarlas, constituyen resultados computables a efectos de la determinación de la renta neta.

Para los efectos de la determinación del Impuesto a la Renta, por operaciones en moneda extranjera, se aplicarán las siguientes normas:

[...]

b) Las diferencias de cambio que resulten del canje de la moneda extranjera por moneda nacional, se considerarán como ganancia o como pérdida del ejercicio en que se efectúa el canje.

c) Las diferencias de cambio que resulten de los pagos o cobranzas por operaciones pactadas en moneda extranjera, contabilizadas en moneda nacional, que se produzcan durante el ejercicio se considerarán como ganancia o como pérdida de dicho ejercicio.

d) Las diferencias de cambio que resulten de expresar en moneda nacional los saldos de moneda extranjera correspondientes a activos y pasivos, deberán ser incluidas en la determinación de la materia imponible del período en el cual la tasa de cambio fluctúa, considerándose como utilidad o como pérdida. [...] ».

La Resolución del Tribunal Fiscal 974-5-2012 establece que: «La naturaleza de las diferencias de cambio no es la de un gasto que deba evaluarse al amparo del principio de causalidad, por lo que para establecer cuál es el alcance del Artículo 61 de la LIR es pertinente analizar qué debe entenderse por operaciones que fuesen objeto habitual de la actividad gravada y las que se produzcan por razones de los créditos obtenidos para financiarlas". Asimismo, la Resolución del Tribunal Fiscal 1003-4-2008 señala que «los efectos de las diferencia por tipo de cambio afectan a la renta neta como ingresos y gastos, sin embargo, al generarse tal diferencia en un ajuste por efecto de la variación del valor de la moneda en el tiempo y no en operaciones realizadas por los contribuyentes a efectos de generar o mantener fuente o rentas gravadas, carecen de la naturaleza de gasto como lo define el Artículo 37 de la LIR, constituyendo en realidad, la diferencia negativa, una pérdida deducible para efectos tributarios». 
Legislativo 1112 publicado el 29 de junio de 2012 y vigente desde el 01 de enero de 2013.

En este sentido, la diferencia en cambio de pasivos relacionados con activos, incurrida en el periodo pre operativo hasta el 31 de diciembre de 2012 debería seguir el tratamiento especial dispuesto en el inciso f) del Artículo 61 de la LIR vigente, y no ser considerada como gasto pre operativos, según el inciso g) del Artículo 37 de la LIR.

\subsection{Existencia de varios proyectos y plazo de amortización.}

Otro tema que puede generar controversia es el relacionado con el tratamiento de los gastos pre operativos en los casos en los que exista en forma paralela varios proyectos, todos ellos en etapa pre operativa.

En efecto, en tales casos surge la duda sobre si la opción prevista en el inciso g) del Artículo 37 de la LIR debería aplicarse uniformemente a los gastos pre operativos de todos los proyectos en curso, o si es posible adoptar tratamientos distintos por cada proyecto; es decir, amortizar en plazos distintos cada proyecto, e incluso deducir íntegramente los gastos en algunos casos mientras otros se amortizan.

Escenarios como este podrían presentarse en el caso de empresas mineras, que desarrollan exploraciones en distintas concesiones en forma paralela.

Cabe precisar que la norma en cuestión no regula en forma expresa el tratamiento en este tipo de escenarios.

En este sentido, y no existiendo prohibición o limitación alguna, creemos que si es posible identificar adecuadamente y sustentar la distinción entre los gastos pre operativos asociados a uno u otro proyecto, no debería existir mayor inconveniente en adoptar distintos tratamientos para los gastos pre operativos generados por cada proyecto. No obstante ello, por un criterio de seguridad jurídica no estaría de más una precisión reglamentaria sobre este asunto.

\subsection{Proyectos Truncos.}

Ocurre en el devenir de la actividad de las empresas que, iniciada la etapa pre operativa de un proyecto y habiéndose realizado ya gastos relacionados con éste, luego de una evaluación económica se llegue a la conclusión que el proyecto no es viable. En ese momento, el proyecto se deja sin efecto.

Los desembolsos realizados hasta ese momento calificarían como gastos pre operativos, por lo que habrían sido acumulados para ser deducidos bajo alguna de las formas que señala el inciso g) del Artículo 37 de la LIR. Pero a partir del momento en que el proyecto se declara trunco, estos gastos dejarían de tener la 
calidad de pre operativos, porque ya no precederían una etapa operativa o de producción.

En este sentido, creemos que los gastos acumulados hasta ese momento deberían ser deducidos para propósitos tributarios en el periodo en que se declara la inviabilidad del proyecto.

En esa misma línea, se ha pronunciado el Tribunal Fiscal mediante la resolución 0591-4-2008 en la cual señala que:

«Al respecto, cabe indicar que tratándose de costos por estudios de obra incurridos en el ejercicio 1999, cuya ejecución no se había iniciado en tal ejercicio, los mismos califican como gastos pre operativos por lo que resulta arreglado a Ley que la recurrente los considere como tal y los registrara como un activo diferido, resultando asimismo razonable que la recurrente, al conocer de la inviabilidad del proyecto, los dedujera como gasto [...]»

Creemos que no podría alegarse que, por no estar dichos gastos conectados con la generación de ingresos gravables, no son deducibles. En efecto, es parte de la vida de las empresas evaluar nuevos emprendimientos que, de resultar exitosos, generarían mayores rentas gravables. En consecuencia, el hecho que dichos proyectos no resulten exitosos no significa per se que los gastos no sean deducibles. 